\title{
A NEW CRITERION FOR THE ROUGHNESS OF EXPONENTIAL DICHOTOMY ON $\mathbb{R}$
}

\author{
O. LEONTIEV AND P. FEKETA
}

Received 26 January, 2015

\begin{abstract}
It is well known (see [Palmer, K.J.: Exponential dichotomies and transversal homoclinic points, J. Differential Equations 55 (1984), 225-256]) that system $x^{\prime}=A(t) x$ possesses exponential dichotomy on $\mathbb{R}^{+}$iff so does perturbed system $x^{\prime}=(A(t)+B(t)) x$, where perturbation $B(t)$ is "small", that is $\lim _{t \rightarrow+\infty}\|B(t)\|=0$. However, it is also known that corresponding statement for exponential dichotomy on $\mathbb{R}$ fails. In this work we show that under additional hypothesis of commutativity of $A(s)$ and $B(t)$ for all $s, t \in \mathbb{R}$ it can indeed be shown that dichotomy on $\mathbb{R}$ is unaffected by "small" perturbations (while "small" now has meaning of "being in $L^{1}(\mathbb{R})$ class"). We also provide some examples and particular corollaries of theory to show that hypotheses are natural and apply to wide range of problems.
\end{abstract}

2010 Mathematics Subject Classification: 34D09; 34D10

Keywords: exponential dichotomy, perturbation theory, linear differential equations

\section{INTRODUCTION}

Consider the system

$$
x^{\prime}(t)=A(t) x(t),
$$

where $x(t)$ and $A(t)$ are respectively vector-valued and square-matrix-valued functions defined for $t \in S \subseteq \mathbb{R}$. In subsequent, we will impose conditions on $A(t)$, so that any initial value problem $x\left(t_{0}\right)=x_{0}, t_{0} \in S$ has a unique continuous solution that extends to the whole $S$. This will allow us to speak of the fundamental matrix $\Omega_{a}^{b}(A)$, which is a linear map that maps $x_{0}$ to $x(b)$, where $x(t)$ satisfies the aforementioned system and initial conditions $x(a)=x_{0}$ (linearity follows from the homogeneity of a system). Now, the system is said to possess "exponential dichotomy" on $S$ (or simply "dichotomy" in subsequent), if the phase space $\mathbb{R}^{n}$ can be decomposed into the direct sum of subspaces $E^{+} \oplus E^{-}$and for arbitrary $x^{+} \in E^{+}, x^{-} \in E^{-}$and $a, b \in S$ the following estimates hold:

$$
\begin{array}{r}
\left\|\Omega_{0}^{b}(A) x^{+}\right\| \leq K\left\|\Omega_{0}^{a}(A) x^{+}\right\| \cdot e^{-\gamma|a-b|}, a \leq b, \\
\left\|\Omega_{0}^{b}(A) x^{-}\right\| \leq K\left\|\Omega_{0}^{a}(A) x^{-}\right\| \cdot e^{-\gamma|a-b|}, b \leq a
\end{array}
$$


for some constants $K, \gamma>0$, that do not depend on $x^{+}, x^{-}, a, b$.

Remark 1. The alternative definition, as given in [3] can be formulated as follows: there exist projection-operator $P$ and numbers $K, \gamma>0$, such that for $\forall a, b \in S$

$$
\begin{gathered}
\left\|\Omega_{0}^{a}(A) P\left(\Omega_{0}^{b}(A)\right)^{-1}\right\| \leq K e^{-\gamma(a-b)}, a \geq b, \\
\left\|\Omega_{0}^{a}(A)(I-P)\left(\Omega_{0}^{b}(A)\right)^{-1}\right\| \leq K e^{-\gamma(b-a)}, b \geq a .
\end{gathered}
$$

The concept of exponential dichotomy plays an important role both in theory and applications. For instance in [1], it has been utilized for the development of sufficient conditions for the existence of mean square bounded solutions to linear stochastic Ito system. In [6], exponential dichotomy properties have been utilized for the investigation of multi-frequency oscillations and qualitative behavior of solutions of linear extensions of dynamical systems on torus. Roughness of exponential dichotomy (that is, its persistence under "small" perturbations) is the main property that allows to apply this concept to the investigations of a variety of real-world phenomena and processes. This causes a particular relevance of research in this field.

One of the first monographs entirely devoted to the subject of exponential dichotomy was the book of Coppel [3]. It contains the exposition of how the dichotomy relates to basic concepts in systems of ODE, such as roughness, Lyapunov functions and functional analysis aspects. It also encompasses the criteria for dichotomy and roughness conditions, in particular that exponential dichotomy on any interval $J$ is not affected when the perturbation $B(t)$ satisfies $\|B\|_{\infty}:=\sup _{t \in J}\|B(t)\|<\delta$, where $\delta$ is some fixed constant, depending on function $A$. From there, this roughness result was worked upon by numerous researchers. For example, Palmer in [9] has shown (among other results) that dichotomy on $\left[t_{0},+\infty\right.$ ) is preserved under perturbation $B(t)$ if $\lim _{t \rightarrow \infty}\|B(t)\|=0$. The constant $\delta$ mentioned above was sharpened in [8], where authors also have extended Coppel's roughness statement to the case when dichotomy constants $K$ and $\gamma$ (see above) can be different for $E^{+}$and $E^{-}$. Besides, in [4], authors tackled the case when $A$ is assumed only locally integrable, not necessarily continuous or even bounded on $J$.

Although in this work we confine ourselves to the finite-dimensional setting, one can easily introduce the corresponding exponential dichotomy theory to the case of operators on Banach spaces with many results and proofs carrying through. Then it is fruitful to study the preservation of dichotomy under "small" (in some sense) perturbations in this new generalised setting. Anyhow, we refer the interested reader to $[2,5,10]$ and references therein.

In this work we show that under additional hypothesis of commutativity of $A(s)$ and $B(t)$ for all $s, t \in \mathbb{R}$ it can indeed be shown that dichotomy on $\mathbb{R}$ is unaffected by "small" perturbations (while "small" now has meaning of "being in $L^{1}(\mathbb{R})$ class"). We also provide some examples and particular corollaries of theory to show that 
hypotheses are natural and apply to wide range of problems. Some comparison with previously known results will follow.

The rest of the paper is organized as follows: In Section 2 we state our main result, Section 3 is devoted to its proof. In Section 4 we have collected some corollaries and examples to highlight how our result relates to the previously known ones. Finally, the last section concludes with a short discussion of a further work.

\section{Results}

In subsequent, all matrix functions will be assumed to be continuous. In particular, continuity assumption will let us immediately assume that Cauchy problem for every ODE system $x^{\prime}=A(t) x$ has a unique solution on $\mathbb{R}$ for any initial conditions.

Theorem 1. Assume that for the two systems

$$
x^{\prime}=A(t) x(t)
$$

and

$$
x^{\prime}=(A(t)+B(t)) x(t)
$$

the following two conditions hold:

(1) For any $t, s \in \mathbb{R}$ matrices $A(s)$ and $B(t)$ commute;

(2) $B(t) \in L^{1}(\mathbb{R}) \cap C(\mathbb{R})$, that is $\int_{-\infty}^{\infty}\|B(s)\| d s<\infty$.

Then, (2.2) has exponential dichotomy on $\mathbb{R}$ if (2.1) does.

Remark 2. The hypothesis of the theorem 1 about the commutativity (the first condition) holds in particular if $\forall t, B(t) \in \bigcap_{s \in \mathbb{R}} Z_{\mathfrak{g l}_{n}}(A(s))$, where $Z_{\mathfrak{g l}_{n}}(A)$ denotes the (Lie algebra) centralizer of $A$. If furthermore $A(t) \equiv A$, this condition can be simply rewritten as $\forall t, B(t) \in Z_{\mathfrak{g l}_{n}}(A)$.

\section{PROOFS}

Proof of Theorem 1. The desired result will follow from two lemmas, which we shall state now and prove later. It should be noted that the lemmas have weaker hypothesis than that of the theorem, and are useful results on their own.

Lemma 1. Let $A(t)$ and $B(t)$ be continuous (not necessary bounded matricesvalued functions on $\mathbb{R})$. Assume further that for every $s, t \in \mathbb{R} A(s)$ and $B(t)$ commute. Then, for the system $x^{\prime}=B(t) x$, every Cauchy problem has a unique solution on $\mathbb{R}$, in other words, the fundamental matrix (we shall denote it by $X_{B}(t)$ ) is welldefined on $\mathbb{R}$ and for arbitrary $t, s \in \mathbb{R}, A(t)$ and $X_{B}(s)$ commute.

Lemma 2. Let $B(t)$ be continuous matrix-valued function, such that $B(t) \in L^{1}(\mathbb{R})$. Then, the fundamental matrix $X_{B}(t)$ of $x^{\prime}=B(t) x$ is well-defined on $\mathbb{R}$, and both $\left\|X_{B}(t)\right\|$ and $\left\|X_{B}^{-1}(t)\right\|$ are uniformly bounded in $t \in \mathbb{R}$. 
Assuming these two for the moment, let us see how the proof of the theorem can be obtained from them. Consider the system $x^{\prime}=B(t) x$, whose fundamental matrix (existing by Lemma 2) we shall denote by $X_{B}(t)$. Let us make the variable change $x=X_{B}(t) z$. Then, we have

$$
B X_{B} z+X_{B} z^{\prime}=\left(X_{B} z\right)^{\prime}=x^{\prime}=(A+B) X_{B} z
$$

Now, as by Lemma $1 A$ and $X_{B}$ commute, we have

$$
X_{B} z^{\prime}=X_{B} A z
$$

thus $z$ is the solution to $z^{\prime}=A z$, hence the fundamental matrix of $x^{\prime}=(A+B) x$ has the structure

$$
X_{A+B}=X_{B} X_{A} .
$$

Now, from [3] we know, that the exponential dichotomy on $\mathbb{R}$ can be defined as the existence of projection operator $P$ and numbers $\gamma, K>0$, such that $\forall s, t \in \mathbb{R}$ we have

$$
\begin{gathered}
\left\|X(t) P X^{-1}(s)\right\| \leq K e^{-\gamma(t-s)}, s \leq t, \\
\left\|X(t)(I-P) X^{-1}(s)\right\| \leq K^{-\gamma(s-t)}, t \leq s .
\end{gathered}
$$

We shall show, that this estimation remains the same for perturbed system 2.2 with the same $P$ and $\gamma$, but perhaps different $K$. Indeed, we shall have

$$
\begin{aligned}
& \left\|X_{A+B}(t) P X_{A+B}^{-1}(s)\right\|=\left\|X_{B}(t) X_{A}(s) P X_{A}^{-1}(t) X_{B}^{-1}(t)\right\| \leq \\
& \leq\left\|X_{B}(t)\right\| \cdot\left\|X_{A}(s) P X_{A}^{-1}(t)\right\| \cdot\left\|X_{B}^{-1}(t)\right\| \leq \underbrace{K C^{2}}_{=: \tilde{K}} e^{-\gamma(t-s)} .
\end{aligned}
$$

The second inequality is worked out in the same way, so it just remains to prove the lemmas stated above.

Proof of Lemma 2. The existence of fundamental matrix follows immediately from the continuity assumption on $B(t)$. The uniform boundedness of $\left\|X_{B}(t)\right\|$ follows from [12, Corollary 3.24] (which states that if the system $x^{\prime}=C x$ with $C$ constant is uniformly stable and $B(t) \in L^{1}\left(\mathbb{R} \cap C(\mathbb{R})\right.$, then $x^{\prime}=(C+B(t)) x$ is stable as well; in our case we take obviously stable system $x^{\prime}=0 \cdot x$ as $\left.x^{\prime}=C x\right)$. We shall denote the upper bound obtained for $X_{B}(t)$ by $M$. It is sufficient to show that $\left\|X_{B}^{-1}(t)\right\| \leq M$. Let us fix $\tau \in \mathbb{R}$. $X_{B}^{-1}(\tau)$ can be characterized as $X_{B}^{-1}(\tau) x(\tau)=$ $x(0)$, where $x$ is the solution to $x^{\prime}=B(t) x$ or as $X^{-1}(\tau) y(0)=y(-\tau)$, where $y^{\prime}=\tilde{B} y$ and $\tilde{B}(t):=B(t+\tau)$. Thus, it's enough to show that the norm of $X_{\tilde{B}}$ is uniformly bounded by $M$ as well. The latter, however, follows from the proof of [12, Corollary 3.24], as the upper bound obtained there really depends only on the $\int_{-\infty}^{\infty}\|\tilde{B}(s)\| d s=\int_{-\infty}^{\infty}\|B(s)\| d s$

Proof of Lemma 1. We shall employ the theory of Magnus expansion, described in [7]. For convenience, we collect the properties we need in the following proposition 
Proposition (Magnus Expansion, see [7]). Assume that we have a system $x^{\prime}=$ $B(t) x$ with continuous $B(t)$ and fundamental matrix $X(t)$. Assume further that for some $T$ we have

$$
\left|\int_{0}^{T}\|B(s)\| d s\right|<\pi
$$

then, on $t \in[0, T) X(t)=\exp (\Omega(t))$, where $\Omega(t)=\sum_{k=1}^{\infty} \Omega_{k}(t)$ and $\Omega_{k}(t)$ is an integral of commutators of increasing length, such as

$$
\begin{aligned}
& \Omega_{1}(t)=\int_{0}^{t} B\left(t_{1}\right) d t_{1}, \\
& \Omega_{2}(t)=\frac{1}{2} \int_{0}^{t} d t_{1} \int_{0}^{t_{1}} d t_{2}\left[B\left(t_{1}\right), B\left(t_{2}\right)\right],
\end{aligned}
$$

$$
\begin{aligned}
\Omega_{3}(t)= & \frac{1}{6} \int_{0}^{t} d t_{1} \int_{0}^{t_{1}} d t_{2} \int_{0}^{t_{2}} d t_{3}\left(\left[B\left(t_{1}\right),\left[B\left(t_{2}\right), B\left(t_{3}\right)\right]\right]+\left[B\left(t_{3}\right),\left[B\left(t_{2}\right), B\left(t_{1}\right)\right]\right]\right), \\
\Omega_{4}(t)= & \frac{1}{12} \int_{0}^{t} d t_{1} \int_{0}^{t_{1}} d t_{2} \int_{0}^{t_{2}} d t_{3} \int_{0}^{t_{3}} d t_{4}\left(\left[\left[\left[B_{1}, B_{2}\right], B_{3}\right], B_{4}\right]\right. \\
& \left.+\left[B_{1},\left[\left[B_{2}, B_{3}\right], B_{4}\right]\right]+\left[B_{1},\left[B_{2},\left[B_{3}, B_{4}\right]\right]\right]+\left[B_{2},\left[B_{3},\left[B_{4}, B_{1}\right]\right]\right]\right)
\end{aligned}
$$

Having this, we shall fix $\tau \in \mathbb{R}$ and show that for any $t \in \mathbb{R}$,

$$
\left[A(\tau), X_{B}(t)\right]=0,
$$

where $[\cdot, \cdot]$ denotes the Lie bracket. As for $t=0$ the equality holds (as $X(0)=I$ commutes with all matrices), it is enough to show that the set where equality holds is both open and closed. As both $A, X$ and Lie bracket are continuous, closedness follows and it remains thus to show, that if $\left[A(\tau), X\left(t_{0}\right)\right]=0$, then equality also holds on a small neighborhood of $t_{0}$.

It is sufficient to show that $A(\tau)$ commutes with $\tilde{X}(s):=X\left(t_{0}+s\right) X^{-1}\left(t_{0}\right)$ for small $s$. Now, $\tilde{X}$ can be realized as a fundamental matrix of $x^{\prime}=\tilde{B}(t) x$, where $\tilde{B}(t)=B\left(t+t_{0}\right)$. Thus, without loss of generality we may assume that $t_{0}=0$. Now, on the small neighborhood of 0 the hypothesis of Proposition is satisfied, hence we may apply Magnus expansion. 
Now, if $A$ and $B$ commute with $C$, then so do $A \pm B, A B$ and $[A, B]$. Also, if $B(t)$ commutes with $A$ for all $t$, then so does $\int_{a}^{b} B(s) d s$. Thus, we see that every $\Omega_{k}(t)$ commutes with $A(\tau)$, as former is the integral of commutators of $B$. Finally, as Lie bracket is continuous, we also have that $\Omega(t)$ commutes with $A(\tau)$ whenever well-defined, and so does $X(t)=\exp (\Omega(t))=\sum_{n=0}^{\infty} \frac{\Omega^{n}(t)}{n !}$.

\section{Corollaries AND EXAMPLes}

We recall the often used in analysis space of test functions, called Schwartz space. These are characterized as

$$
\left\{f \in C^{\infty}(\mathbb{R})\left|\forall \alpha, \beta \in \mathbb{Z}, \quad \sup _{x}\right| x^{\alpha} D^{\beta} f(x) \mid<\infty\right\} .
$$

The definition can be simply adapted to matrix-valued functions. Schwartz space is easily shown to be strictly bigger than the more often used space of $C^{\infty}(\mathbb{R})$ functions with compact support. As it is a well-known fact that Schwartz functions are $L^{1}(\mathbb{R})$, we have the following.

Corollary 1. Let $x^{\prime}=A(t) x(t)$ be the system with an exponential dichotomy on $\mathbb{R}$, $B(t)$ be the matrix valued Schwartz function, such that $\forall s, t \in \mathbb{R} A(t)$ and $B(s)$ commute. Then the perturbed system $x^{\prime}=(A(t)+B(t)) x(t)$ also possesses exponential dichotomy.

As Schwartz functions are so commonly encountered nowadays in analysis, we hope this corollary to be of some interest and use.

Another way to produce interesting corollaries may be on contrary, to strengthen commutativity hypothesis of Theorem 1 . In this direction the following may be of interest

Corollary 2. If $x^{\prime}=c x$ is system with exponential dichotomy, where $c$ is a (scalar) constant (that is the same as require $\operatorname{Re} c \neq 0$ ), then for any matrix-valued $L^{1}(\mathbb{R})$ function $B(t)$ perturbed system has also dichotomy.

It should be noted that this corollary is not a direct corollary of well-known result [12, Corollary 3.21]. Indeed, that result states that asymptotical stability is unaffected by $o(1)$-perturbations (that is, such that $\lim _{t \rightarrow \pm \infty}\|B(t)\|=0$ ), while previous corollary states that it is unaffected by $L^{1}(\mathbb{R})$ perturbations (indeed, for $x^{\prime}=c x$ exponential dichotomy is the same as forward/backward asymptotic stability). As neither of classes $o(1)$ and $L^{1}(\mathbb{R})$ is not a subset of other, last corollary is no direct implication of [12, Corollary 3.21].

To conclude, we shall demonstrate one example, illustrating how in some particular cases our derived results allow to make more precise conclusions on dichotomy behaviour and persistence than the original results of Palmer in [9] or Coppel in [3]. 
Example 1. Consider the two-dimensional system

$$
\left[\begin{array}{c}
\dot{x} \\
\dot{y}
\end{array}\right]=\left[\begin{array}{cc}
1 & 1 \\
0 & -1
\end{array}\right]\left[\begin{array}{l}
x \\
y
\end{array}\right] \text {. }
$$

The eigenvalues of the matrix are real, so system has a dichotomy on $\mathbb{R}$. Now, perturb it to get

$$
\left[\begin{array}{c}
\dot{x} \\
\dot{y}
\end{array}\right]=\left[\begin{array}{cc}
1+P(t) e^{-t^{2}} & 1+(P-Q)(t) e^{-t^{2}} / 2 \\
0 & -1+Q(t) e^{-t^{2}}
\end{array}\right]\left[\begin{array}{l}
x \\
y
\end{array}\right],
$$

where $P(t)$ and $Q(t)$ are arbitrary polynomials in $t$.

Note, that both the original matrix $\left[\begin{array}{cc}1 & 1 \\ 0 & -1\end{array}\right]$ and perturbation we've applied $\left[\begin{array}{cc}P(t) & (P-Q)(t) / 2 \\ 0 & Q(t)\end{array}\right] e^{-t^{2}}$ commute. Then, our theorem shows that perturbed system still possess the dichotomy on $\mathbb{R}$ (as we've perturbed by Schwartz function, see above), while Palmer's theorem only allows one to conclude that the dichotomy on $\pm \mathbb{R}$ is preserved. Neither Coppel's classical result from [3], nor any of the latter developments $([4,8])$ cannot be applied for general $P$ and $Q$, as any of them requires the supremum-norm $\|B(t)\|_{\infty}$ of perturbation be not bigger than some particular value, while evidently by adjusting $P$ and $Q$ latter can be made arbitrarily big.

\section{FURTHER WORK}

The theorem we have proven together with its corollaries listed can be possibly extended to other classes of differential equations, whenever the concept of exponential dichotomy is of relevance to them. In particular new results and corresponding proof techniques could be applied to the investigations of the existence of invariant toroidal manifolds of multi-frequency systems, for which the exponential dichotomy plays an essential role (see $[6,11]$ for details).

\section{ACKNOWLEDGEMENT}

The authors express their gratitude to Professor Mykola Perestyuk for valuable comments and discussion.

\section{REFERENCES}

[1] A. M. Ateiwi, "About bounded solutions of linear stochastic Ito systems," Miskolc Mathematical Notes, vol. 3, pp. 3-12, 2002.

[2] L. Barreira and C. Valls, "Smooth robustness of exponential dichotomies," Proceedings of the American Mathematical Society, vol. 139, no. 3, pp. 999-1012, 2011, doi: 10.1090/S0002-99392010-10531-2.

[3] W. Coppel, Dichotomies in stability theory, ser. Lecture Notes in Mathematics. Springer, 1978.

[4] N. Ju and S. Wiggins, "On roughness of exponential dichotomy," Journal of Mathematical Analysis and Applications, vol. 262, no. 1, pp. 39 - 49, 2001, doi: 10.1006/jmaa.2001.7496.

[5] N. Lupa and M. Megan, "Exponential dichotomies of evolution operators in Banach spaces," Monatshefte fur Mathematik, vol. 174, no. 2, pp. 265-284, 2014, doi: 10.1007/s00605-013-0517-y. 
[6] Y. Mitropolsky, A. Samoilenko, and V. Kulik, Dichotomies and Stability in Nonautonomous Linear Systems, ser. Stability and Control: Theory, Methods and Applications. Taylor \& Francis, 2002.

[7] P. C. Moan and J. Niesen, "Convergence of the Magnus series," Found. Comput. Math., vol. 8, no. 3, pp. 291-301, Jun. 2008, doi: 10.1007/s10208-007-9010-0.

[8] R. Naulin and M. Pinto, "Admissible perturbations of exponential dichotomy roughness," Nonlinear Analysis: Theory, Methods \& Applications, vol. 31, no. 5-6, pp. 559 - 571, 1998, doi: 10.1016/S0362-546X(97)00423-9.

[9] K. J. Palmer, "Exponential dichotomies and transversal homoclinic points," Journal of Differential Equations, vol. 55, no. 2, pp. 225-256, 1984, doi: 10.1016/0022-0396(84)90082-2.

[10] L. H. Popescu, "Exponential dichotomy roughness on Banach spaces," Journal of Mathematical Analysis and Applications, vol. 314, no. 2, pp. 436 - 454, 2006, doi: 10.1016/j.jmaa.2005.04.011.

[11] A. Samoilenko, Elements of the Mathematical Theory of Multi-Frequency Oscillations, ser. Mathematics and its Applications. Springer Netherlands, 1991.

[12] G. Teschl, Ordinary Differential Equations and Dynamical Systems, ser. Graduate Studies in Mathematics. American Mathematical Society, 2012.

Authors' addresses

O. Leontiev

The University of Tokyo, Graduate School of Mathematical Sciences, 3-8-1 Komaba Meguro-ku Tokyo, 153-8914 Tokyo, Japan

E-mail address: leontiev@ms.u-tokyo.ac.jp

P. Feketa

University of Applied Sciences Erfurt, Department of Civil Engineering, Altonaer Str. 25, 99085 Erfurt, Germany

E-mail address: petro.feketa@fh-erfurt.de 